\title{
NOUVELLE
}

\section{Les agrégats amyloïdes de la protéine prion HET-s sont infectieux}

Marie-Lise Maddelein

\section{Le concept}

\section{d'une « protéine infectieuse »}

L'idée qu'une protéine puisse être infectieuse a émergé en 1967 des études réalisées par T. Alper et J. Griffith sur la tremblante, une encéphalopathie spongiforme transmissible (EST) du mouton [1]. En 1982, Stanley Prusiner tente de purifier l'agent infectieux responsable de la tremblante. Sa composition essentiellement protéique lui inspire le terme de proteinaceous infectious element, en abrégé: prion. C'est cette hypothèse d'une protéine seule responsable des EST (protein only hypothesis) qui lui a valu le prix Nobel de médecine en 1997.

Or, si de nombreux arguments sont venus renforcer cette hypothèse, l'existence de protéines infectieuses n'a toujours pas été confirmée. Le rôle essentiel de la protéine prion des mammifères, la $\operatorname{Pr} P$, dans les EST, a été démontré par l'obtention de souris délétées de ce gène et devenues résistantes envers cette maladie [2]. Cependant, les tentatives utilisant la $\operatorname{Pr} P$ recombinante (produite dans $\varepsilon$. coli) pour induire l'apparition de la tremblante sont restées sans succès [3]. Ces résultats n'excluent pas que la protéine PrP puisse être l'agent infectieux des maladies à prions des mammifères et, d'ailleurs, aucun autre candidat n'a pour l'instant été identifié.

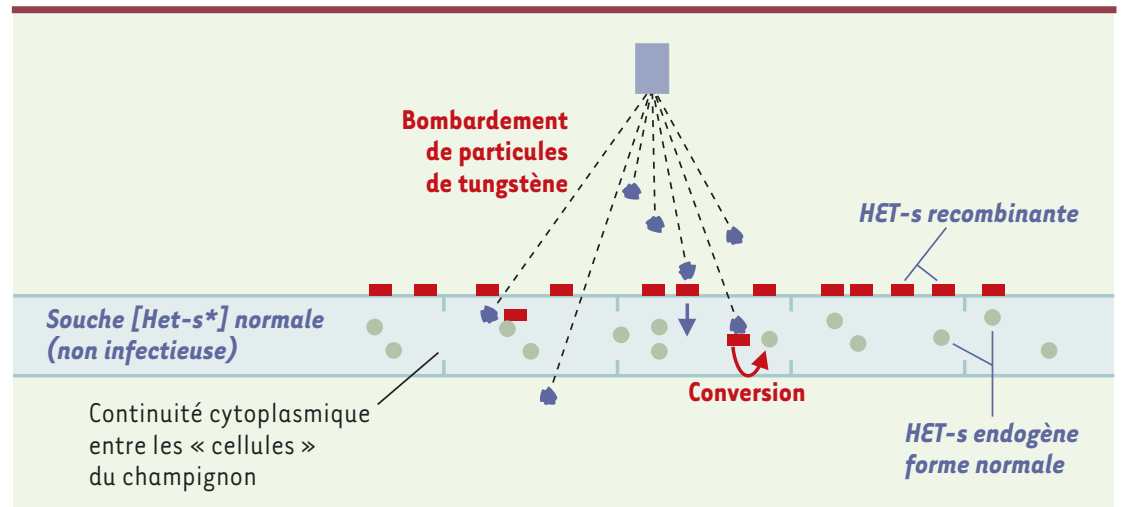

Figure 1. Principe de l'essai biolistique. Nous avons adapté cette technique de biolistique afin de contrôler l'état d'agrégation de la protéine: celle-ci est déposée sur le mycélium et les bombardements de particules de tungstène (bleu) introduisent la protéine recombinante (rouge) dans le champignon. La souche de départ, de phénotype neutre [Het-s*] (non infectieuse), exprime la protéine HET-s sous forme soluble dans le cytoplasme [8]. Après bombardement, des particules de tungstène peuvent être visualisées dans le mycélium et l'introduction de la protéine HET-s recombinante contrôlée par marquage à la fluorescéine [7]. Conversion: modification de la protéine cellulaire normale en forme infectieuse. La forme infectieuse [Het-s] se propage ensuite à toute la souche grâce à la communication cytoplasmique entre les « cellules » du champignon.

Les études génétiques effectuées dans les micro-organismes sont venues confirmer l'existence d'une hérédité protéique. En 1994, Reed Wickner démontre que les éléments de génétique non mendélienne [URE3] et [PSI] de la levure Saccharomyces cerevisiae se comportent comme des prions [4]. II établit alors les critères génétiques permettant d'identifier un caractère héréditaire lié à la présence d'un prion dans les micro-organismes.

Sur la base de ces critères, le groupe de Joël Bégueret a alors identifié l'élément particulier [Het-s] du champignon filamenteux Podospora anserina comme étant un prion [5]. Le caractère [Het-s] est infectieux car il se transmet par simple contact à une souche normale appelée [Het-s*]. Le caractère [Het-s] peut apparaître spontanément et il se propage alors rapidement à tout le champignon. Comme pour les prions de la levure, le caractère [Het-s] peut être « guéri » de façon réversible par certains traitements. De plus, [Het-s] est strictement sous la dépendance de l'expression du gène codant pour la protéine HET-s dont la surexpression induit systématiquement l'émergence du caractère infectieux [Het-s].

\section{La démonstration formelle qu'une protéine peut être infectieuse}

La preuve ultime du caractère infectieux d'une protéine peut être obtenue si l'introduction de la protéine recombinante purifiée dans un organisme est suffisante pour propager le caractère infectieux [6]. Nous avons purifié la protéine recombinante HET-s et testé différentes méthodes pour l'introduire dans Podospora. L'avantage de la technique de la biolistique, décrite sur la Figure 1, est de permettre l'introduction de molécules dans 
des mycéliums entiers. En effet, le mycélium des champignons filamenteux présente une continuité cytoplasmique favorisant la propagation d'éléments infectieux. Ainsi, même si l'introduction de la protéine recombinante est un événement rare, l'apparition de la forme infectieuse [Het-s] dans un article (cellule non cloisonnée du mycélium) doit se transmettre à la protéine HET-s endogène et envahir rapidement tout le champignon (Figure 1). Grâce à cette continuité cytoplasmique, l'essai biolistique s'est montré très efficace: l'introduction de la protéine recombinante HET-s a permis d'induire le caractère infectieux [Het-s] dans presque $100 \%$ des souches neutres [Het-s*] [7]. Nous avons ainsi démontré clairement le caractère infectieux de la protéine recombinante HET-s de Podospora et, par là même, confirmé l'existence de protéines infectieuses ou prions. tique ont été identifiés comme des agrégats amyloïdes [9]. Visualisés par microscopie électronique, ces agrégats sont essentiellement composés de fibrilles. Les fibres amyloïdes sont connues pour résister à la digestion par les protéases. Nous avons observé que les agrégats de HET-s restent infectieux même après traitement à la protéinase K. L'introduction de la protéine recombinante HET-s dénaturée (par la chaleur ou par l'acide) n'induit pas l'apparition du caractère [Het-s]. Lorsque la protéine HદT-s est dénaturée, elle forme des agrégats amorphes en microscopie électronique et ceux-ci semblent alors dépourvus de pouvoir infectieux.

$\varepsilon$ n définitive, nos résultats montrent que les fibres amyloïdes produites in vitro sont capables de servir de modèle à la réplication du prion in vivo. De plus, nous avons observé que les agrégats de la protéine HET-s native (formés dans le champignon) sont capables de convertir la protéine HET-s recombinante soluble en agrégats amyloïdes [7]. Ces résultats suggèrent que des agrégats amyloïdes peuvent exister in vivo $[10,11]$.

Les données corrélant l'agrégation amyloïde et le caractère infectieux des protéines prions s'accumulent, confirmant que ces deux phénomènes sont intimement liés. Cependant, la nature moléculaire du matériel infectieux in vivo reste encore à préciser.

Le protocole expérimental décrit ici, constitue un essai biologique très sensible qui pourrait nous permettre de tester d'autres protéines ou des peptides potentiellement infectieux. Cet essai biolistique utilisant le champignon pourrait également être appliqué à la caractérisation des protéines prions provenant d'autres espèces, et en particulier à la PrP des mammifères. $\diamond$

HET-s gene product behaves as a prion

\section{Seuls les agrégats}

\section{amyloïdes}

sont infectieux

Les protéines prions présentent une propriété commune aux protéines amyloïdes: celle de former des agrégats in vivo, et des fibres amyloïdes in vitro. Dans la levure et Podospora, l'acquisition du caractère infectieux semble corrélée à l'agrégation de la protéine prion in vivo $[1,8]$.

Dans notre essai biolistique, la forme infectieuse de la protéine recombinante HET-s est strictement associée à son état insoluble (Figure 2). La protéine HET-s soluble isolée par ultracentrifugation n'induit pas l'apparition du phénotype infectieux [Het-s]. Les agrégats de la protéine HET-s utilisés en biolis-

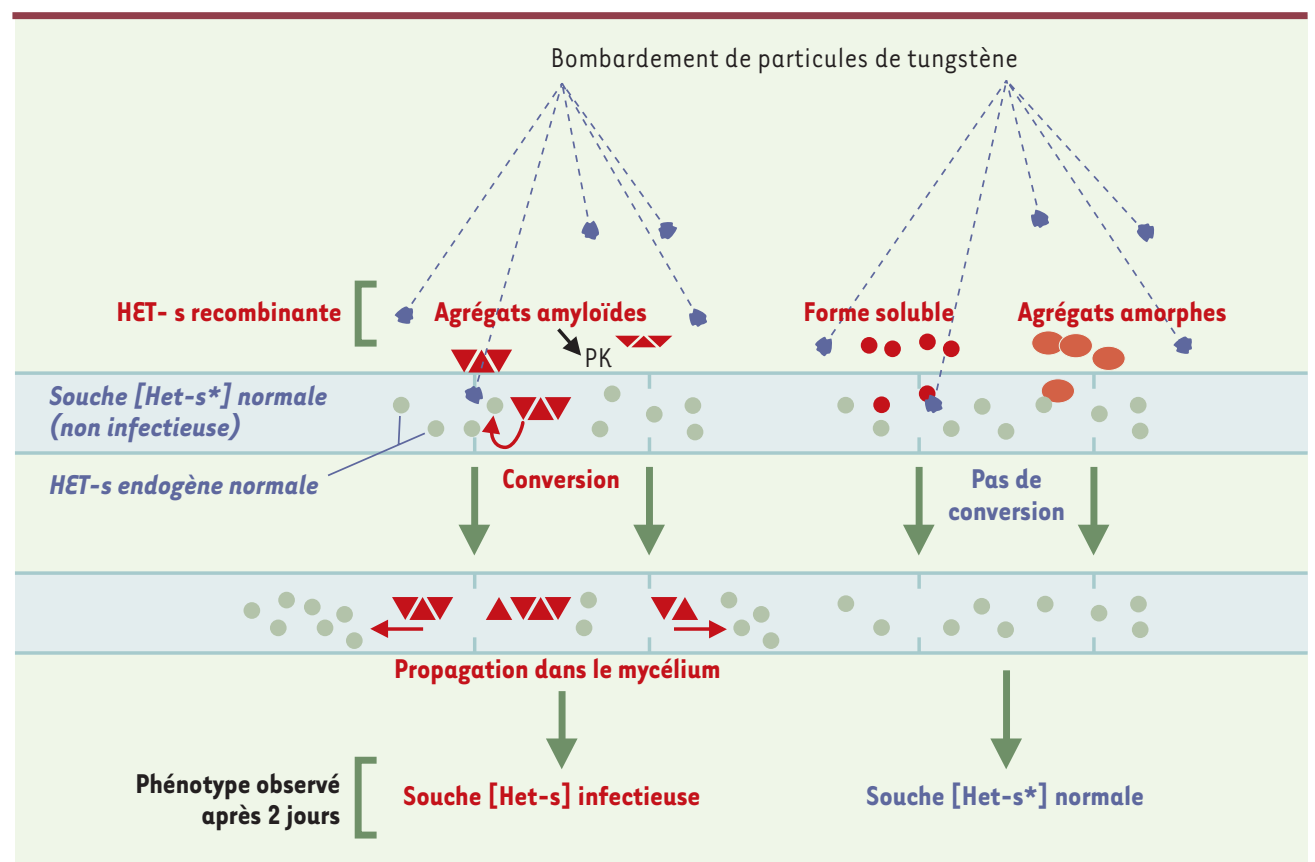

Figure 2. La forme infectieuse de la protéine recombinante HET-s dépend de son état d'agrégation. Après renaturation, la protéine HET-s recombinante s'agrège spontanément en fibres amyloïdes résistantes à la digestion par la protéinase K (PK) [9]. L'introduction de ces fibres amyloïdes dans une souche neutre [Het-s*] induit systématiquement le phénotype infectieux [Het-s]. L'apparition de ce phénotype démontre que la protéine introduite est capable de convertir la protéine cellulaire normale en forme infectieuse [7]. L'introduction de la protéine recombinante HET-s sous forme soluble (séparée des agrégats par ultracentrigation) ou dénaturée par la chaleur ou l'acide (agrégats amorphes) n'induit pas le phénotype infectieux [Het-s]. 


\section{REMERCIEMENTS}

Nos recherches sont soutenues par le Cnrs, la Fondation pour la Recherche Médicale, et le Ministère de la Recherche (GIS prion).

\section{RÉFÉRENCES}

1. Wickner RB, Taylor KL, Edskes HK, Maddelein ML, Moriyama H, Roberts BT. Prions in Saccharomyces and Podospora spp.: protein-base inheritance. Microbiol Mol Biol Rev 1999; 63: 844-61.

2. Bueler HM, Aguzzi A, Sailer $A$, et al. Mice devoid of PrP are resistant to scrapie. Cell 1993; 73: 1339-47.

3. Caughey B. Transmissible spongiform

encephalopathies, amyloidoses and yeast prions: common threads? Nat Med 2000; 6: 751-4.

4. Wickner RB. Evidence for a prion analog in

S. cerevisae: the [URE3] non-Mendelien genetic element as an altered URE2 protein. Science 1994; 264 : 566-9.

5. Coustou V, Deleu C, Saupe $\mathrm{S}$, Bégueret J. The protein product of the het-s heterocaryon incompatibility gene of the fungus Podospora anserina behaves as a prion analog. Proc Natl Acad Sci USA 1997; 94 : 9773-8.

6. Liautard JP, Alvarez-
Martinez MT, Féraudet C, Torrent J. La protéine prion: structure, dynamique et conversion in vitro. Med Sci 2002; 18: 62-9.

7. Maddelein ML, Dos Reis S, Duvezin-Caubet $S$, CoularySalin B, Saupe S. Amyloid aggregates of the HET-s prion protein are infectious. Proc Natl Acad Sci USA 2002; 99 : 7402-7.

8. Coustou-Linares V, Maddelein ML, Bégueret J, Saupe S. In vivo aggregation of the HET-s prion protein of the fungus Podospora anserina. Mol Microbiol 2001; 42: 1325-35.

9. Dos-Reis S, Coulary-Salin B, Forges V, Lascu I,
Bégueret J, Saupe S. The HET-s prion protein of the filamentous fungus Podospora anserina aggregates in vitro into amyloid-like fibrils. J Biol Chem 2002; 277 : 5703-6.

10. Uptain SM, Sawinicki GJ, Caughey $B$, Lindquist S. Strains of $[\mathrm{PSI}(+)]$ are distinguished by their efficiencies of prionmediated conformational conversion. EMBO J 2001 ; $20: 6236-45$.

11. Speransky VV, Taylor KL, Edskes HK, Wickner RB, Steven AC. Prion filament networks in [URE3] cells of Saccharomyces cerevisiae. J Cell Sci 2001; 153: 1327-36.

\section{Une protéine chaperon pour la synthèse coordonnée des chaînes de globine}

Dominique Labie

> L'hémoglobine $(\mathrm{Hb})$ est un tétramère dont les deux chaînes $\alpha$ et $\beta$ sont codées par des gènes situés sur des chromosomes différents. Une coordination est nécessaire pour assurer un équilibre stœchiométrique parfait entre les deux processus de synthèse. L'excès de l'une ou de l'autre chaîne se traduira, en effet, par un syndrome thalassémique plus ou moins sévère. Les chaînes $\beta$ en excès forment des homotétramères $\beta^{4}$ $(\mathrm{HbH})$, inaptes au transport de l'oxygène, mais solubles; les chaînes $\alpha$, en revanche, ont tendance à précipiter dans la cellule sous forme monomérique, entraînant une cascade physiopathologique dont l'étape finale est la dénaturation de la protéine et la destruction cellulaire prématurée, phénomènes lar- gement responsables des troubles observés au cours des $\beta$-thalassémies [1]. Les études de synthèse de I'Hb ont montré qu'en raison d'un léger excès de synthèse des chaînes d' $\alpha$ globine par rapport aux chaînes de $\beta$ globine, il ne se forme pas spontanément d'HbH. Le mécanisme évitant les désordres dus à l'excès de chaînes $\alpha$ a fait l'objet d'hypothèses non vérifiées, dont l'existence d'une protéine «chaperon » assurant leur stabilisation. L'article publié dans Nature par deux équipes de l'Université de Philadelphie (PA, USA) semble apporter une réponse à cette question non résolue [2].

Les auteurs sont partis de l'hypothèse que cette protéine stabilisatrice devait avoir, comme les chaînes de globine et les enzymes de la synthèse de l'hème, une spécificité érythroïde, et devait donc être contrôlée par GATA-1, facteur de transcription à doigts de zinc indispensable à la spécification de la lignée érythroïde [3]. Le criblage des gènes dont la synthèse était induite par GATA-1 leur a permis d'isoler un ARNm transcrit à partir d'un gène appelé EDRF (erythroid differentiation-related factor) qu'ils proposent d'appeler plutôt AHSP ( $\alpha$-haemoglobin stabilizing factor). L'ARN murin de ce gène EDRF/AHSP code pour une protéine de 102 acides aminés, très conservée à travers les espèces, abondante et de spécificité érythroïde rigoureuse, très diminuée dans les cultures de cellules GATA-1. Les auteurs ont ensuite mis en évidence une liaison spécifique de la protéine AHSP avec l' $\alpha$ globine monomérique, et pas avec la $\beta$ - 